\title{
Key role of dual specificity kinase TTK in proliferation and survival of pancreatic cancer cells
}

\author{
B P Kaistha ${ }^{1}$, T Honstein ${ }^{1}$, V Müller ${ }^{1}$, S Bielak ${ }^{1}$, M Sauer ${ }^{1}$, R Kreider ${ }^{1}$, M Fassan ${ }^{2}$, A Scarpa ${ }^{2}$, C Schmees $^{3}$, \\ H Volkmer ${ }^{3}$, T M Gress ${ }^{1}$ and M Buchholz ${ }^{*, 1}$ \\ ${ }^{1}$ Department of Medicine, Division of Gastroenterology, Endocrinology and Metabolism, Philipps University Marburg, \\ Marburg, Germany; ${ }^{2}$ Dipartimento ad Attività Integrata (DAl) di Patologia e Diagnostica, University of Verona, Verona, Italy and \\ ${ }^{3}$ Department of Molecular Biology, Natural and Medical Sciences Institute of the University of Tübingen, Reutlingen, \\ Tübingen, Germany
}

Background: Pancreatic ductal adenocarcinoma (PDAC) is among the most aggressive human malignancies with an overall 5-year survival rate of $<5 \%$. Despite significant advances in treatment of the disease during the past decade, the median survival rate ( $\sim 6$ months) has hardly improved, warranting the need to identify novel targets for therapeutic approaches.

Methods: Quantitative real time PCR, western blot analyses and immunohistochemical staining of tissue microarrays were used to analyse the expression of TTK gene in primary PDAC tissues and cell lines. To inhibit TTK kinase expression in a variety of pancreatic cancer cell lines, RNA interference was used. Functional roles of this kinase in the context of PDAC were studied using cell proliferation, viability and anchorage-independent growth assays. Western blotting, fluorescence-activated cell sorting analyses and fluorescence microscopy were used to gain mechanistic insight into the functional effects.

Conclusions: We show that the dual specificity kinase TTK (also known as Mps1), is strongly overexpressed in human PDAC. Functionally, cell proliferation was significantly attenuated following TTK knockdown, whereas apoptosis and necrosis rates were significantly increased. In addition, anchorage-independent growth, a hallmark of malignant transformation and metastatic potential, was strongly impaired in the absence of TTK gene function. Interestingly, immortalised normal pancreatic hTERT-HPNE cells were not affected by loss of TTK function. Mechanistically, these effects in cancer cells were associated with increased formation of micronuclei, suggesting that loss of TTK function in pancreatic cancer cells results in chromosomal instability and mitotic catastrophe. Taken together, our data show that TTK function is critical for growth and proliferation of pancreatic cancer cells, thus establishing this kinase as an interesting new target for novel therapeutic approaches in combating this malignancy.

Pancreatic ductal adenocarcinoma (PDAC) is one of the most aggressive solid cancers and presently the fourth leading cause of cancer-related deaths in the western world (Hidalgo, 2010; Jemal et al, 2011; Malvezzi et al, 2013). Currently, PDAC is practically incurable with existing therapies. The vast majority of the patients are diagnosed at an advanced and often already metastasised stage, making curative resection impossible. Both, incidence and mortality are on the rise for PDAC, unlike for other cancer types. For the European Union, the death rate in pancreatic cancer is expected to rise from 7.86 in 2007 to 8.0 per 100000 among men, and from 5.24 to 5.5 per 100000 among women in 2013, and the trend is predicted to continue (Malvezzi et al, 2013). The median survival rate ( $\sim 6$ months) and the overall 5-year survival rate $(<5 \%)$ has not improved despite significant research and diagnostic efforts over the 
last decade, illustrating the urgent need to identify novel molecular targets and approaches to combat this devastating disease.

Protein kinases are critical regulators of diverse cellular functions. They are now widely acknowledged as effective druggable targets in a variety of pathological conditions. The chemistry and pharmacology of kinase inhibitors have also developed tremendously over the last two decades (Cohen, 2002), enabling most of the inhibitors to be available orally. Consequently, we have performed high-throughput screens for protein kinases, which are upregulated in pancreatic cancer and, which, when silenced, would impair the viability of pancreatic cancer cells.

TTK (also known as Mps1) is a dual specificity kinase, that is, it can act to phosphorylate both tyrosine as well as serine/threonine residues (Liu and Winey, 2012). First identified in budding yeast, it is highly conserved from yeast to humans (Mills et al, 1992; Liu and Winey, 2012). It is regarded as a key player in several steps of mitosis in a variety of eukaryotic cells (Liu et al, 2003) and is one of the main kinases involved in kinetochore localisation and the spindle assembly checkpoint (SAC), which inhibits metaphase-toanaphase transition until proper spindle attachment to all chromosomes is achieved (Stucke et al, 2002; Liu and Winey, 2012; Nijenhuis et al, 2013). Several reports have indicated the role of TTK in a variety of disease patterns (Poss et al, 2002; Liu and Winey, 2012; Althoff et al, 2012; Morin et al, 2012), including, in an apparent paradox to its role as a checkpoint kinase, a growth promoting role in cancer cells of different origin. Here, we show for the first time that TTK is significantly overexpressed in pancreatic cancer and that it has a central role in maintaining the viability and proliferative potential of pancreatic cancer cells.

\section{MATERIALS AND METHODS}

Cell lines and primary tissues. The human pancreatic adenocarcinoma cell lines Panc1, PaTu-8988 T and S2-028 were used in this study. Pancl cells were obtained from the German Collection of Microorganisms and Cell Cultures (DSMZ, Braunschweig, Germany). S2-028 cells were from T Iwamura (Miyazaki Medical College, Miyazaki, Japan) (Taniguchi et al, 1992). PaTu-8988 $\mathrm{T}$ cells were kindly provided by $\mathrm{H} \mathrm{P}$ Elsässer (Cytobiology and Cytopathology Institute, Philipps University, Marburg, Germany). These cancer cell lines were maintained in Dulbecco's modified minimal essential medium (GIBCO, Grand Island, NY, USA) supplemented with 10\% FCS (PAA, Cölbe, Germany) and cultured at $37^{\circ} \mathrm{C} / 5 \% \quad \mathrm{CO}_{2}$. The non-transformed pancreatic cell line hTERT-HPNE was kindly provided by C Schmees, NMI, Reutlingen, Germany. The basal medium for these cells consisted of $75 \%$ DMEM without glucose (GIBCO) with an additional $2 \mathrm{~mm}$ L-glutamine and $1.5 \mathrm{gl}^{-1}$ sodium bicarbonate and $25 \% \mathrm{M} 3$ Medium (Incell Corp., San Antonio, TX, USA). For experiments, complete growth medium was used, which additionally contained $5 \%$ FCS, $10 \mathrm{ng} \mathrm{ml}^{-1}$ human recombinant EGF (Sigma, Germany), $5.5 \mathrm{~mm} \mathrm{D}$-glucose and $750 \mathrm{ng} \mathrm{ml}^{-1}$ puromycin (PAA). Immortalised human pancreatic ductal cells (HPDE) (Ouyang et al, 2000) were cultured in Defined Keratinocyte Serum-Free medium supplemented with human recombinant epidermal growth factor and bovine pituitary extract (Gibco/Life Technologies, Darmstadt, Germany).

Cancer cell line identities were verified using the GenomeLab Human STR Primer Set (Beckman Coulter, Krefeld, Germany) on a CEQ8800 sequencer (Beckman Coulter) according to the manufacturer's protocol. To confirm the identity of human cell lines, STR data were submitted to online verification tool of DSMZ (www.dsmz.de).

Surgically resected PDAC and chronic pancreatitis tissue samples were procured from the surgery departments of the
University of Heidelberg and Ludwig Maximilian University of Munich. Samples of normal pancreatic tissue were obtained from the healthy donors. Informed consent in writing was obtained from all patients prior to using tissue samples. The study was approved by the ethics committee at the University of Heidelberg and Ludwig Maximilian University of Munich, Germany.

siRNA transfection of cell lines. PaTu-8988T, Panc1 and S2-028 cells were transfected with siRNAs using siLentFect Lipid Reagent (Bio-Rad, Munich, Germany) according to the manufacturer's protocol. Unless stated otherwise, for transfection, $150 \times 10^{3}$ cells were seeded per well in a 6-well plate. Three specific siRNAs targeting TTK gene were used: Hs_TTK_2, Hs_TTK_6 and Hs_TTK_7 (FlexiTube, Qiagen, Hilden, Germany); henceforth only distinguished as si1, si2 and si3, respectively. Accell Control siRNA \#1 (Thermo Scientific-Dharmacon, Lafayette, CO, USA) was used as a non-silencing control ( $\mathrm{siC}$ ). Untreated cells, henceforth designated NT, were used in all experiments as additional control.

RNA extraction, cDNA sysnthesis and qRT-PCR. RNA from cell lines was extracted using the peqGold Total RNA Kit (PEQLAB $\mathrm{GmbH}$, Erlangen, Germany) according the manufacturer's protocol. Tissue samples were homogenised in liquid nitrogen using a mortar and pestle. Extraction of RNA was done using the RNeasy Mini Kit (Qiagen) following the manufacturer's protocol. One microgram total RNA was used for first-strand cDNA synthesis using the Omniscript RT Kit (Qiagen) as per the manufacturer's protocol. Quantitative real time reverse transcription PCR (qRT-PCR) was performed using SYBR Green MasterMix (Applied Biosystems, Foster City, CA, USA) on a 7500 Fast Realtime PCR system (Applied Biosystems). The following primer pairs were used for qRT-PCR: ribosomal protein, large, P0; RPLP0_For: 5'-TGGGCAAGAACACCATGATG-3'; RPLP0_Rev: 5'-AGTTTCTCCAGAGCTGGGTTGT-3'; TTK_For: 5'-TGGCCAACCTGCCTGTTT-3'; TTK_Rev: 5'-AATGCATT CATTTGCTGAAGAAGA-3'.

Protein extraction and western blotting. For protein extraction, cells were collected together with medium and centrifuged at $1600 \mathrm{rpm}$ at $4{ }^{\circ} \mathrm{C}$ for $5 \mathrm{~min}$. Pellets were washed twice with ice-cold PBS and then resuspended in $200 \mu$ l lysis buffer (PBS containing protease inhibitors (Protease Arrest, GBiosciences, St Louis, MO, USA) and phosphatase inhibitors (PMSF $1 \mathrm{~mm}$, EDTA $0.5 \mathrm{~mm}$, sodium pyrophosphate $25 \mathrm{~mm}$, sodium orthovanadate, $10 \mathrm{~mm}$, sodium luoride $50 \mathrm{~mm}$ )). Cells were sonicated (LabSonic, BBraun, Melsungen, Germany) and protein content was assessed using Protein Assay Reagent (Thermo Scientific). For western blotting, $10 \mu \mathrm{g}$ proteins were electrophoresed on SDS-polyacrylamide gels and electrophoretically transferred onto nitrocellulose membranes (Optitran, GE Healthcare Life Sciences, Little Chalfont, UK). Membranes were blocked in 5\% non fat dry milk in TBST (10 mM Tris- $\mathrm{HCl}, \mathrm{pH}$ 7.6, $100 \mathrm{~mm} \mathrm{NaCl}, 0.1 \%$ Tween 20 ) for $2-4$ h at room temperature and then probed with appropriate primary and secondary antibodies (anti-Phospho-Chk1 (Ser345): Cell Signaling, Boston, MA, USA; cat\# 2341; anti-Phospho-Histone H2A.x (Ser139): Cell Signaling, cat\# 9718; anti-Cyclin B1: Cell Signaling, cat\# 4135; anti-Mps1 (TTK): Millipore; cat\# 05-682; anti-PARP: Cell Signaling, cat\# 9532 and; anti-Caspase-3: Cell Signaling, cat\# 9664 and 9665).

Immunohistochemistry. Two tissue microarrays (TMAs) including a retrospective series of 40 FFPE surgically treated PDACs and 8 non-neoplastic controls were retrieved from the ARC-Net biobank at Verona University Hospital. Four 1-mm-tissue cores per case were analysed. The materials have been collected under Program 853 protocol 298CE 15/02/02 and revised Program 1885 protocol 52438 $23 / 11 / 2010$, approved by the Verona University Hospital ethics committee. The protocols include informed consent from the patient. 
Immunohistochemical stainings for TTK (clone 3-472-1; Upstate Biotechnology, Lake Placid, NY, USA; $1: 100$ ) were obtained on $4-\mu$ m-thick FFPE sections using an automated instrument (BondmaX, Menarini, Rome, Italy). Sections were lightly counterstained with hematoxylin. Appropriate positive and negative controls were run concurrently. TTK cytoplasmic immunostaining was considered positive. In all the considered tissue samples, granulocytes and endothelia always featured TTK cytoplasmic expression and were assumed as positive internal control (not retained for IHC scoring).

BrdU cell proliferation assay. DNA synthesis as a direct measure of cell growth was measured using the Cell Proliferation ELISA, BrdU chemiluminescence Kit (Roche, Mannheim, Germany) according to the manufacturer's protocol. Briefly, 5000 cells were re-seeded $24 \mathrm{~h}$ after transfection into a 96-well plate (ViewPlate Black Clear Bottom, PerkinElmer, Rodgau, Germany). On the next day, cells were incubated for $4 \mathrm{~h}$ with BrdU-containing medium, then fixed at room temperature for $1 \mathrm{~h}$ and incubated with the peroxidase-conjugated anti-BrdU antibody for $1.5 \mathrm{~h}$. The chemiluminescence intensity was measured as relative light units per second $\left(\right.$ rlu s$\left.^{-1}\right)$.

MTT cell viability and cell survival assay. 24 hours post transfection, $30 \times 10^{3}$ cells per well were re-seeded into 12 -well plates. After additional $48 \mathrm{~h}$ of incubation, cells were incubated for $1.5 \mathrm{~h}$ with MTT-containing medium prepared by dissolving thiazolyl blue (Carl Roth, Karlsruhe, Germany). The cells were then solubilised using 10\% Triton X-100 (Carl Roth)/0.1 M HCl (Fisher Scientific, Schwerte, Germany) in isopropanol (SigmaAldrich, Taufkirchen, Germany) and the extinction was then measured at $570 \mathrm{~nm}$ on a plate reader. For the cell survival assay, $10 \times 10^{3}$ cells were seeded per well in seven 24 -well plates and transfected with siRNAs next day. Over a period of 7 days, MTT assays were performed and cell survival was plotted as relative cell number by measuring the MTT extinction coefficient for each day.

Soft agar assay. Soft agar clonogenic assays were performed in a 12 -well plate format as described before (Hann et al, 2011). Briefly, $6 \times 10^{3}$ siRNA-transfected cells were re-seeded in a $0.33 \%$ DMEMbacto-agar per well onto a bottom layer of 0.5\% DMEM-bactoagar. One microlitre DMEM medium was added after the top agar had solidified. Medium was replaced every third day. Quantification was done by counting the number of colonies formed after 10 days of culturing.

Flow cytometric analysis. For the cell cycle analyses, $48 \mathrm{~h}$ after transfection, cells were collected, washed with PBS and then were fixed overnight at $4{ }^{\circ} \mathrm{C}$ with ice-cold $70 \%$ ethanol. Fixed cells were washed again once with ice-cold PBS and incubated with FxCycle Violet Stain (1:1000, Molecular Probes, Invitrogen, Darmstadt, Germany) for $30-45 \mathrm{~min}$ in the dark. Measurement was done at the central fluorescence-activated cell sorting (FACS) facility of the Medical Faculty, Marburg University, on a FACS LSR-II (BD Biosciences, Heidelberg, Germany) machine by acquiring a minimum of 20000 cells in the Pacific Blue channel. For the Annexin-V/Propidium iodide (PI) staining, $48 \mathrm{~h}$ after transfection, the cells were collected, washed with PBS, resuspended in the binding buffer (10 mM HEPES/NaOH pH7.4, $140 \mathrm{~mm} \mathrm{NaCl}, 2,5 \mathrm{~mm}$ $\mathrm{CaCl}_{2}$ ) and then stained with a FITC-coupled-Annexin- $\mathrm{V}$ antibody (BD Biosciences, cat\# 556419) for $15 \mathrm{~min}$ in the dark. Shortly before measurement, PI was added to the cells. A minimum of $20 \times 10^{3}$ cells were acquired for the experiment at the FACS LSR-II machine.

Fluorescence microscopy. $24 \mathrm{~h}$ after transfection, $20 \times 10^{3}$ cells per well were re-seeded onto coverslips coated with collagen (type I, Sigma-Aldrich) in 12-well plates. After additional $24 \mathrm{~h}$ of incubation, the coverslips were washed with PBS twice, fixed on microscope slides using DAPI containing mounting medium
(Vectashield, Vector Laboratories, Burlingame, CA, USA) and observed under the microscope.

Statistical analysis. Each experiment was repeated independently at least three times. Values are expressed as mean \pm s.e.m. of the triplicates, unless stated otherwise. Student's $t$-test was used to analyse the difference between samples of interest $v s$ control. A $P$-value of $<0.05$ was considered to be statistically significant.

\section{RESULTS}

TTK kinase is upregulated in pancreatic cancer. The mRNA levels of the TTK gene in primary pancreatic cancer and chronic pancreatitis tissues were determined using qRT-PCR and compared with the levels in healthy pancreas samples. A total of 11 cancer tissues, 9 chronic pancreatitis and 8 healthy pancreatic tissues were analysed. A modest increase in the mRNA expression of TTK was seen in chronic pancreatic tissues as compared with the healthy tissues (Figure 1A), whereas pancreatic cancer tissues showed significant overexpression of TTK compared with both chronic pancreatitis as well as healthy pancreatic tissues (Figure 1A). TTK mRNA expression was also readily detectable in all pancreatic cell lines tested, including transformed (IMIM PC1 \& PC2, S2-007, S2-028, PaTu-8988 T and Panc1) as well as non-transformed (HPDE and HPNE) lines. However, no systematic difference in expression levels was observed between cancer $v s$ the normal (immortalised) cell lines (Figure 1B). These findings were further validated by analysing TTK protein levels in primary human tissues as well as cultured cells. Immunohistochemical detection of TTK was performed on TMAs comprising a series of 40 different pancreatic cancer lesions. In normal pancreatic parenchyma, TTK expression was negative/faint in both pancreatic acini and duct epithelia; Langerhans islets showed a moderate TTK expression. A significant overexpression was observed in PDAC lesions. Twenty-eight out of 40 (70\%) PDACs showed a moderate/ strong cytoplasmic immunoreaction in the epithelial cancer cells (Figure 1C). In congruence to the mRNA data, no systematic difference was seen in the TTK protein levels in the normal human pancreatic cell line (HPNE) vs the pancreatic cancer cell lines used for the functional experiments (Figure 1D).

TTK function is indispensible for pancreatic cancer cell proliferation and viability. To assess the functional relevance of this upregulation of TTK in pancreatic cancer, the kinase was transiently silenced using three different specific siRNAs. All three siRNAs led to significant downregulation of the TTK gene product at both transcriptional and protein levels (Figure 2A) in all the three pancreatic cancer cell lines tested (Panc1, S2-028 and PaTu8988T). Downregulation of TTK mRNA after siRNA transfection was measurable as early as $24 \mathrm{~h}$ post transfection and remained stable for over $96 \mathrm{~h}$ (data not shown).

We next used BrdU incorporation assays to analyse the impact of a loss of TTK expression on the proliferative potential of pancreatic cancer cells. In all three cell lines tested, TTK knockdown by the three independent siRNAs led to a significant reduction in proliferation rates compared with cells treated with non-silencing control siRNA (Figure 2B), with the exception of si3 in Pancl cells, where the proliferation was also lower but did not reach statistical significance. These results were confirmed in MTT assays, where knockdown of TTK expression with any of the three independent siRNAs in any of the three pancreatic cancer cell lines led to statistically highly significant decrease in the viability of the cells as compared with controls (Figure 2C). Interestingly, transient loss of TTK function in the non-transformed human pancreatic cell line hTERT-HPNE had no effect on the viability of these cells despite reaching very good knockdown efficiencies 


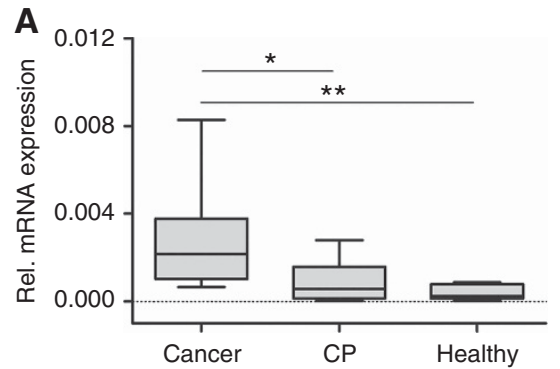

C

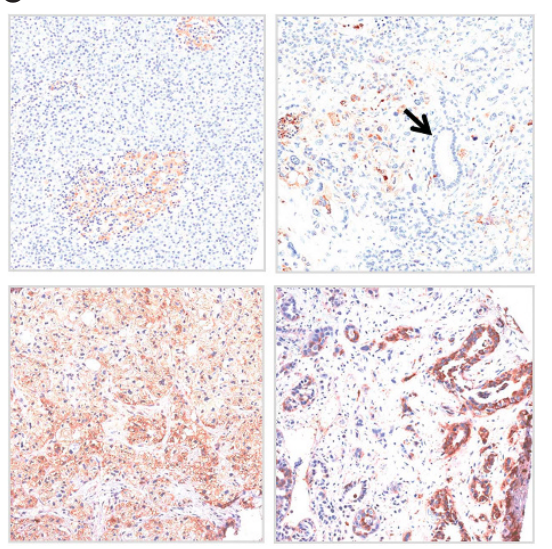

B

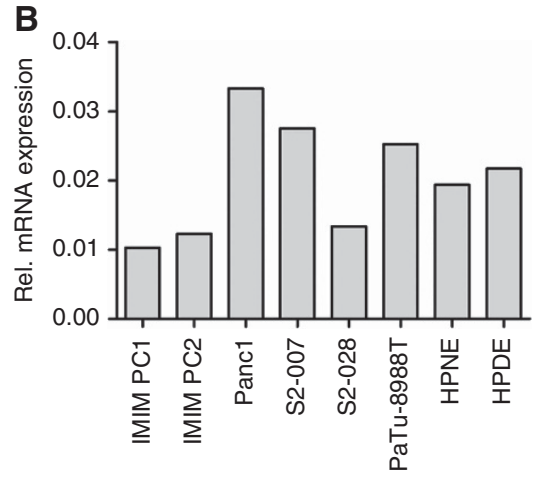

D
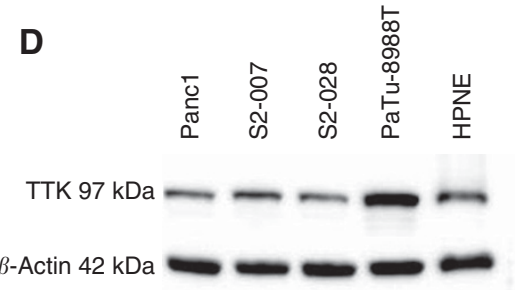

Figure 1. TTK expression in primary pancreatic tumour tissues and cell lines. TTK mRNA and protein levels were examined in primary pancreatic cancer and control tissues as well as in cultured pancreatic cancer and control cell lines. (A) Box-and-whisker plot showing TTK mRNA expression in primary human pancreatic tumour tissue samples, chronic pancreatitis and normal pancreas as analysed by quantitative real time reverse transcription PCR (qRT-PCR). Expression was normalised to ribosomal protein, large, PO (RPLPO) mRNA levels. Data in figure represent median and 2nd and 3rd quartiles (boxes) as well as minimum and maximum values (whiskers). $\mathrm{CP}=$ Chronic pancreatitis. ${ }^{*} P<0.05$; ${ }^{\star *} P<0.01$ (Student's t-test). (B) TTK mRNA expression levels in a variety of different pancreatic cancer and control cell lines. (C) Immunohistochemical staining of tissue microarrays (TMA) for TTK expression. In normal pancreatic tissue, Langerhans islets were stained positive for TTK, whereas normal acinar and ductal cells showed only very faint signal (top left panel). Enhanced expression was seen in the PDAC tissues (top right as well as bottom panels). Normal ducts stained negative for TTK (see arrow, top right panel). (D) Western blot analyses showing TTK expression in the different pancreatic cancer and control cell lines used for the functional experiments. Beta-actin served as loading control.

(Supplementary Figure $1 \mathrm{~A}$ and B), indicating that dependency on TTK expression is cancer cell specific.

Long-term survival studied using the PaTu-8988T cell line demonstrated that efficient and sustained growth inhibition persisted over a period of 7 days following TTK knockdown, thus providing no indication of compensatory mechanisms that would allow cells to successfully escape growth inhibition (Figure 2D).

The ability to continuously proliferate in the absence of adhesion to a solid matrix is a hallmark feature of malignantly transformed epithelial cells and correlates directly with high invasive and metastatic potential. Soft agar assays with Panc1 and S2-028 cells with and without transient TTK knockdown demonstrated that anchorage-independent growth was severely attenuated in the absence of TTK expression in both cell lines (Figure 2E).

TTK knockdown induces apoptotic and necrotic cell death. Western blot analyses of pancreatic cancer cells with and without RNAi-mediated knockdown of TTK expression demonstrated strongly increased PARP (Figure 3A) and/or Caspase-3 (Figure 3B) cleavage in TTK knockdown cells, indicating strongly increased levels of apoptosis in the absence of TTK expression. Moreover, flow cytometry analysis of cells after annexin-V/PI staining further corroborated the western blot findings by demonstrating a strong increase in the numbers of early (annexin-V signal only) and late (annexin-V plus PI signal) apoptotic cells as well as necrotic cells (PI staining only) (Figure 3C) as compared with untreated or control siRNA-treated cells, indicating that cell death can occur via different routes.
Loss of TTK function causes chromosomal missegregation, but not DNA damage or cell cycle arrest in pancreatic cancer cells. Flow cytometric cell cycle analyses of PaTu-8988T cells revealed only a modest influence of TTK knockdown on the relative distribution of cells across different phases of the cell cycle, thus producing no indication of induction of a cell cycle arrest following loss of TTK function (Figure 4A). Concurrently, western blot experiments revealed no evidence of induction of the cellular DNA damage response (DDR). Phosphorylation of the ATR/p53 targets H2AX and Chk1 was not changed, and Cyclin B1 levels were not systematically altered in TTK knockdown cells (Figure 4B), indicating that mitotic exit and cell cycle progression were not attenuated following TTK knockdown.

However, fluorescence microscopy of DAPI-stained cell nuclei demonstrated that genomic integrity was severely compromised in cells lacking functional TTK. In addition to an overall tendency towards increased abnormal nuclear morphology, formation of large numbers of micronuclei, a hallmark feature of massive aneuploidy and chromosomal missegregation, was readily apparent in all three cell lines examined (Figure 5).

\section{DISCUSSION}

Despite considerable efforts, the biology of pancreatic cancer is not clearly understood, and this malignancy continues to pose a significant challenge to clinicians and researchers alike. To date, 

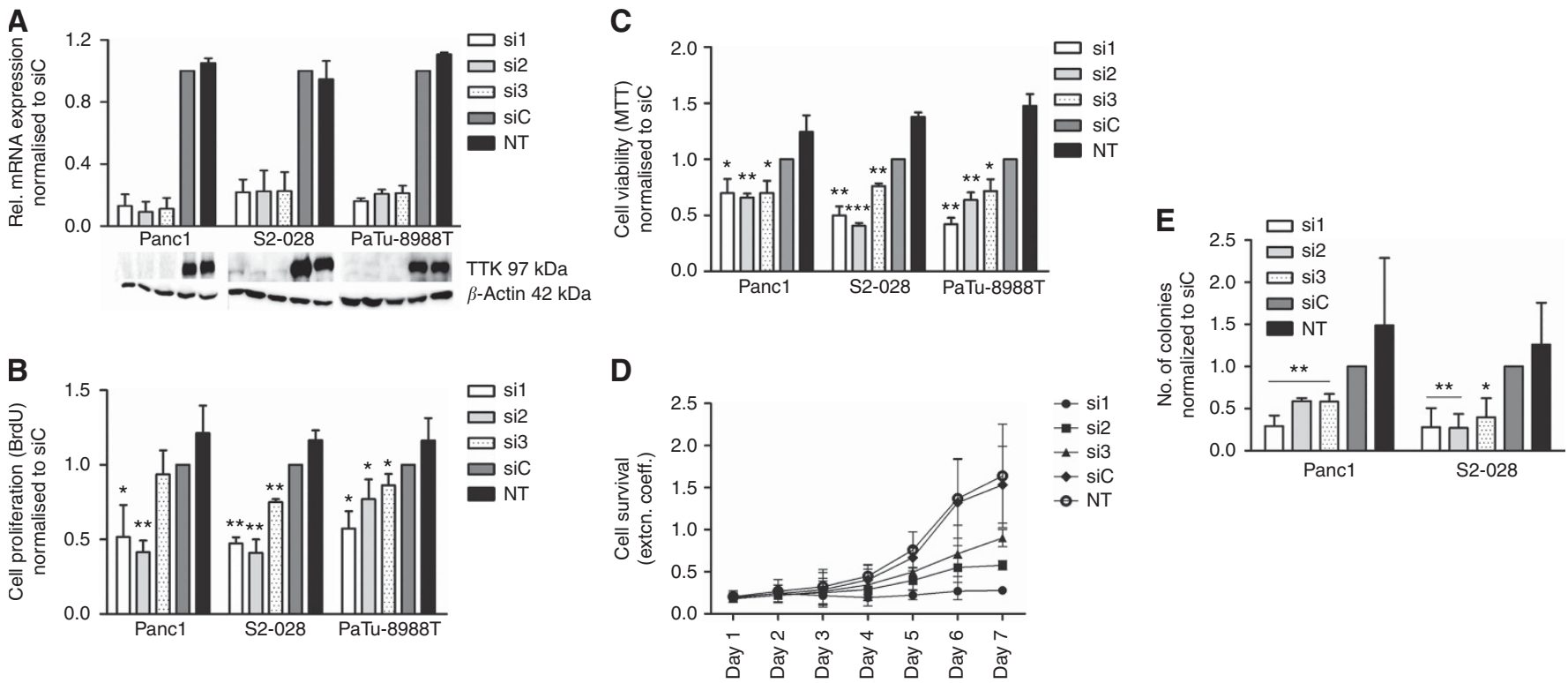

Figure 2. Impaired proliferation and viability of pancreatic cancer cells following RNAi-mediated inhibition of TTK expression. (A) Relative levels of TTK mRNA and protein after transient transfection of TTK-specific and control siRNAs. qRT-PCR analyses demonstrated that all three TTKspecific siRNAs led to $\sim 70 \%$ reduction in TTK mRNA levels compared with non-silencing control siRNA in three different pancreatic cancer cell lines (bar graphs). Expression was normalised to control siRNA-treated cells. Similarly, TTK protein levels were nearly undetectable $72 \mathrm{~h}$ after siRNA transfection as determined by western blot analyses (lower panels). Beta-actin served as loading control. Loss of TTK protein significantly impaired proliferation and viability of pancreatic cancer cell lines as measured by DNA BrdU incorporation (B) and MTT assays (C), respectively. (D) Longterm growth inhibition following transient silencing of TTK in PaTu-8988T cells was stable over at least 7 days as assessed by survival assay. (E) Soft agar assays established significantly reduced numbers of colonies in TTK knockdown populations of Panc1 and S2-028 cells compared with controls after 10 days of continuous culture under non-adherent conditions. All data represent mean \pm s.e.m. of at least three independent experiments. siC $=$ cells transfected with non-silencing control siRNA; NT = untreated cells. ${ }^{\star} P<0.05 ;{ }^{\star \star} P<0.01$; ${ }^{\star \star \star} P<0.001$ (Student's t-test).
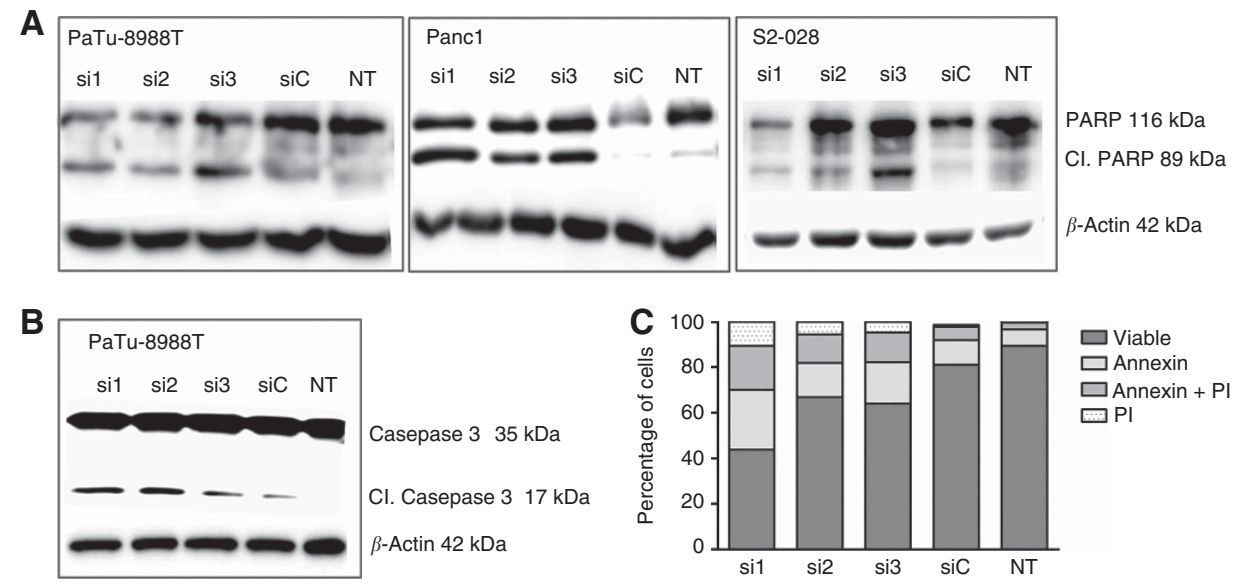

Figure 3. TTK knockdown leads to apoptotic and necrotic death of pancreatic cancer cells. Western blot analysis for induction of apoptosis showed enhanced PARP (A) and/or Caspase-3 (B) cleavage $72 \mathrm{~h}$ after siRNA transfection in all three pancreatic cancer cell lines used in the experiments. Data are representative of at least three independent experiments. Beta-actin served as loading control. (C) Flow cytometry analyses via Annexin-V/PI staining in PaTu-8988 T cells revealed significantly increased proportions of early (Annexin-V signal only) and late (Annexin-V plus PI signal) apoptotic cells as well as necrotic cells (PI staining only) following transient TTK knockdown. Data represent an average of three experiments. $\mathrm{siC}=$ cells transfected with non-silencing control siRNA; NT = untreated cells.

erlotinib, a small molecule inhibitor of EGFR pathway, remains the only targeted therapeutic substance that has been demonstrated to be effective in a subset of pancreatic cancer patients. There is thus a pressing need to identify new targets for novel approaches to the therapy of PDAC. In this context, we focussed on protein kinases, which are especially well suited for pharmacological inhibition, in an effort to identify novel candidates with specific overexpression and essential functional roles in PDAC.
In this report, we show that TTK is overexpressed in human pancreatic cancer tissues as compared with normal pancreas or chronic pancreatitis. Our results are in conformity with previous reports from high-throughput analyses listing TTK among the genes with increased expression in PDAC and mucinous neoplasms of the pancreas, respectively (Grutzmann et al, 2004; Sato et al, 2004). At present, it remains unclear though if TTK expression is a prognostic factor in pancreatic cancer. Moreover, 
A

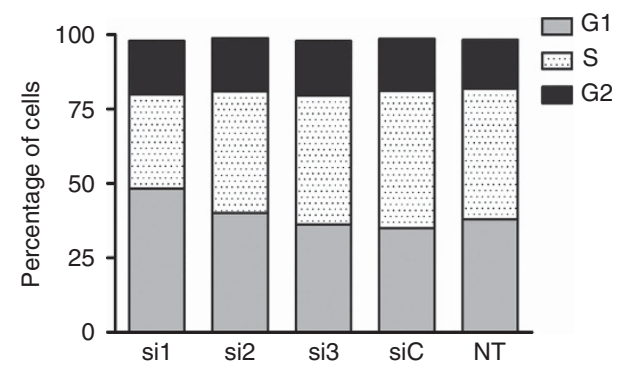

B

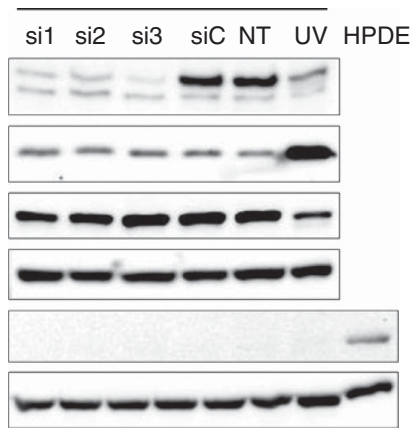

PaTu-8988T

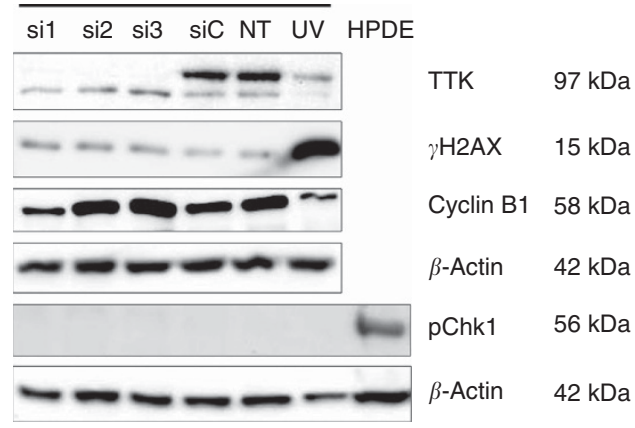

Figure 4. No evidence for cell cycle arrest or DNA damage following TTK knockdown. Cell cycle analysis was done after transient inhibition of TTK expression using FxCycle Violet Stain and flow cytometry of PaTu-8988T cells. TTK inhibition did not result in consistently altered cell cycle distribution (A). DNA damage, as assessed by western blot analyses using phospho-specific antibodies, did not reveal increased phosphorylation levels of the ATR/p53 targets H2AX and Chk1 after TTK silencing. Likewise, cellular Cyclin B1 levels were also not systematically altered (B). UV-treated cancer cells ('UV') served as positive controls for $\gamma \mathrm{H} 2 \mathrm{AX}$ detection; UV-treated normal human pancreatic ductal epithelial (HPDE) cells were used as positive controls for phospho-Chk1 detection. Beta-actin was used as a loading control in these experiments. siC $=$ cells transfected with non-silencing control siRNA; NT=untreated cells.
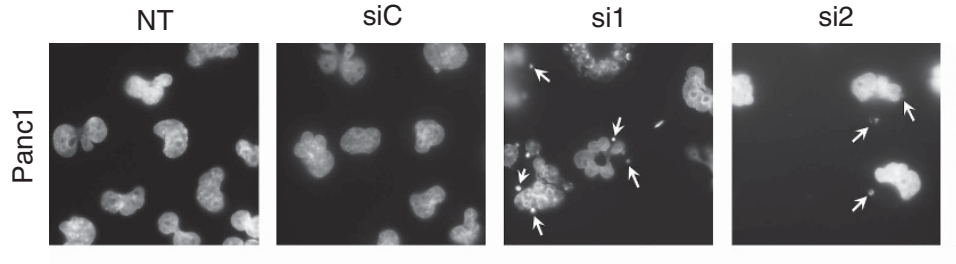

si3
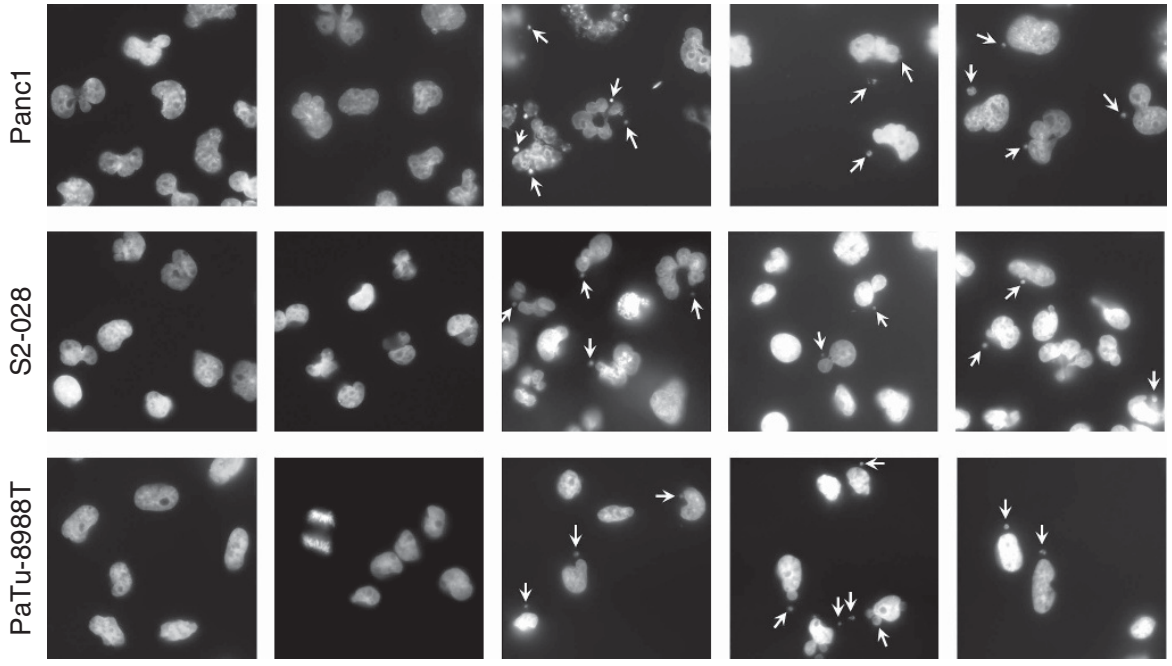

Figure 5. Loss of TTK function leads to genomic instability. Fluorescence microscopy was done using DAPI after transient knockdown of TTK expression. Microscopic analysis revealed formation of micronuclei (white arrows) in all the three cell lines tested, indicating genomic instability and chromosomal missegregation.

the functional significance of TTK overexpression in pancreatic cancer has previously not been analysed.

TTK is an essential component of the SAC, which in normal cells functions to ensure that anaphase is not initiated until each sister chromatid pair is correctly attached to the mitotic spindle (Stucke et al, 2002; Liu and Winey, 2012; Morin et al, 2012). The SAC thus serves as a safeguard against chromosome missegregation and subsequent aneuploidy. In an apparent contradiction to its role as a checkpoint protein, TTK has been shown to be overexpressed in breast cancer and other solid cancers (Yuan et al, 2006; Salvatore et al, 2007; Daniel et al, 2011; Tao et al, 2012), and RNAi-mediated or pharmacological inhibition of TTK function efficiently attenuated growth of a variety of cancer cell lines (Colombo et al, 2010; Tardif et al, 2011). Our own results demonstrate that this also holds true for pancreatic cancer. RNAimediated silencing of TTK gene expression significantly inhibited proliferation and viability of three different pancreatic cancer cell lines as measured by BrdU and MTT assays, strongly induced cell death by both, apoptotic as well as necrotic pathways, and significantly attenuated anchorage-independent growth, a hallmark feature of malignantly transformed epithelial cells. Intriguingly, TTK inhibition seems to affect non-transformed cell types to a much lesser degree. In a report by Daniel et al (2011), transient silencing of TTK decreased viability of the breast cancer cells, but did not inhibit growth of the non-malignant MCF10A cell line. Similarly, Colombo et al (2010) describe that pharmacological inhibition of TTK function reduced viability of a variety of cancer cell lines, but not of normal cells. Our own experiments confirm this observation, as the immortalised but non-transformed hTERT-HPNE cell line was practically unaffected by transient silencing of TTK. Similar results have also been shown for other SAC components such as Mad2 and BubR1 (Kops et al, 2004; Michel et al, 2004; Janssen et al, 2009; Li et al, 2010).

As varying degrees of chromosomal aberrations are commonly observed in most cancers, it can thus be assumed that cancer cells require SAC functions to sustain growth and proliferation in the presence of low levels of aneuploidy. In addition, defects in other checkpoint functions are likely to contribute to the selective 
sensitivity of transformed cells towards SAC inhibition. In normal cells, aneuploidy induces an ATR/p53 response, which results in cell cycle arrest at both, G1/S and G2/M boundaries, and thus prevents propagation of cells with improper chromosomal content (Toledo et al, 2008; Thompson and Compton, 2010). However, the p53 tumour suppressor is mutationally inactivated or deleted in many human cancers, including $50-75 \%$ of pancreatic tumours (Buchholz and Gress, 2009). Accordingly, RNAi-mediated TTK downregulation was not associated with phosphorylation of typical ATR/p53 targets such as H2AX and Chk1 in our cells, and flow cytometric analyses produced no evidence of a common cell cycle arrest phenotype across the individual siRNAs used in the experiments.

In contrast, $\mathrm{H} 2 \mathrm{AX}$ phosphorylation was readily observed following exposure of the cells to UV light, demonstrating that part of the DDR machinery is still intact in the pancreatic cancer cells. At the same time, this implies that, contrary to what has been observed for MCF7 breast cancer and SW480 colon cancer cells (Janssen et al, 2011), SAC inhibition in pancreatic cancer cells does not result in appreciable levels of DNA damage. Instead, it appears that complete undamaged chromosomes were missegregated, as evidenced by the frequent formation of micronuclei after mitosis, which were readily detectable in TTK knockdown cells. This is in agreement with observations in A2780 ovarian cancer and HCT colon cancer cells, where TTK inhibition caused an overall increase in cellular $\gamma \mathrm{H} 2 \mathrm{AX}$ levels, but did not result in DNA damage and formation of $\gamma \mathrm{H} 2 \mathrm{AX}$ foci (Colombo et al, 2010; Tardif et al, 2011). As a final consequence, chromosomal segregation errors ultimately lead to massive aneuploidy, which is incompatible with cellular survival, inducing cell death by either apoptotic mechanisms or mitotic catastrophe.

Taken together, our results demonstrate that pancreatic cancer cells critically depend on the presence of functionally intact SAC machinery. As a kinase that is overexpressed in pancreatic tumours and represents an integral component of the SAC in pancreatic cancer cells, TTK thus appears to be an attractive target for the development of novel therapeutic strategies against this deadly disease. Additional antitumour potency may be gained by a combination with spindle poison-based chemotherapeutics such as nab-Paclitaxel, which is an emerging new standard in the treatment of pancreatic cancer patients.

\section{ACKNOWLEDGEMENTS}

This work funded in part by the German federal ministry of education and research (BMBF) within the framework of the 'KMU-innovativ-4' project 'PakaNostra' (project ID 0315643 C and F) as well as the Italian Association for Research on Cancer (AIRC n. 12182) and the Italian FIMP-Ministry of Health (CUP_J33G13000210001).

\section{CONFLICT OF INTEREST}

The authors declare no conflict of interest.

\section{REFERENCES}

Althoff F, Karess RE, Lehner CF (2012) Spindle checkpoint-independent inhibition of mitotic chromosome segregation by Drosophila Mps1. Mol Biol Cell 23: 2275-2291.

Buchholz M, Gress TM (2009) Molecular changes in pancreatic cancer. Expert Rev Anticancer Ther 9: 1487-1497.

Cohen P (2002) Protein kinases-the major drug targets of the twenty-first century? Nat Rev Drug Discov 1: 309-315.
Colombo R, Caldarelli M, Mennecozzi M, Giorgini ML, Sola F, Cappella P, Perrera C, Depaolini SR, Rusconi L, Cucchi U, Avanzi N, Bertrand JA, Bossi RT, Pesenti E, Galvani A, Isacchi A, Colotta F, Donati D, Moll J (2010) Targeting the mitotic checkpoint for cancer therapy with NMS-P715, an inhibitor of MPS1 kinase. Cancer Res 70: 10255-10264

Daniel J, Coulter J, Woo JH, Wilsbach K, Gabrielson E (2011) High levels of the Mps1 checkpoint protein are protective of aneuploidy in breast cancer cells. Proc Natl Acad Sci USA 108: 5384-5389.

Grutzmann R, Pilarsky C, Ammerpohl O, Luttges J, Bohme A, Sipos B, Foerder M, Alldinger I, Jahnke B, Schackert HK, Kalthoff H, Kremer B, Kloppel G, Saeger HD (2004) Gene expression profiling of microdissected pancreatic ductal carcinomas using high-density DNA microarrays. Neoplasia 6: 611-622.

Hann A, Gruner A, Chen Y, Gress TM, Buchholz M (2011) Comprehensive analysis of cellular galectin-3 reveals no consistent oncogenic function in pancreatic cancer cells. PLoS One 6: e20859.

Hidalgo M (2010) Pancreatic cancer. N Engl J Med 362: 1605-1617.

Janssen A, Kops GJ, Medema RH (2009) Elevating the frequency of chromosome mis-segregation as a strategy to kill tumor cells. Proc Natl Acad Sci USA 106: 19108-19113.

Janssen A, van der BM, Szuhai K, Kops GJ, Medema RH (2011) Chromosome segregation errors as a cause of DNA damage and structural chromosome aberrations. Science 333: 1895-1898.

Jemal A, Bray F, Center MM, Ferlay J, Ward E, Forman D (2011) Global cancer statistics. CA Cancer J Clin 61: 69-90.

Kops GJ, Foltz DR, Cleveland DW (2004) Lethality to human cancer cells through massive chromosome loss by inhibition of the mitotic checkpoint. Proc Natl Acad Sci USA 101: 8699-8704.

Li M, Fang X, Baker DJ, Guo L, Gao X, Wei Z, Han S, van Deursen JM, Zhang P (2010) The ATM-p53 pathway suppresses aneuploidy-induced tumorigenesis. Proc Natl Acad Sci USA 107: 14188-14193.

Liu ST, Chan GK, Hittle JC, Fujii G, Lees E, Yen TJ (2003) Human MPS1 kinase is required for mitotic arrest induced by the loss of CENP-E from kinetochores. Mol Biol Cell 14: 1638-1651.

Liu X, Winey M (2012) The MPS1 family of protein kinases. Annu Rev Biochem 81: 561-585.

Malvezzi M, Bertuccio P, Levi F, La VC, Negri E (2013) European cancer mortality predictions for the year 2013. Ann Oncol 24: 792-800.

Michel L, az-Rodriguez E, Narayan G, Hernando E, Murty VV, Benezra R (2004) Complete loss of the tumor suppressor MAD2 causes premature cyclin B degradation and mitotic failure in human somatic cells. Proc Natl Acad Sci USA 101: 4459-4464.

Mills GB, Schmandt R, McGill M, Amendola A, Hill M, Jacobs K, May C, Rodricks AM, Campbell S, Hogg D (1992) Expression of TTK, a novel human protein kinase, is associated with cell proliferation. J Biol Chem 267: $16000-16006$.

Morin V, Prieto S, Melines S, Hem S, Rossignol M, Lorca T, Espeut J, Morin N, Abrieu A (2012) CDK-dependent potentiation of MPS1 kinase activity is essential to the mitotic checkpoint. Curr Biol 22: 289-295.

Nijenhuis W, von CE, Littler D, De M V, Tromer E, Vleugel M, van Osch MH, Snel B, Perrakis A, Kops GJ (2013) A TPR domain-containing N-terminal module of MPS1 is required for its kinetochore localization by Aurora B. J Cell Biol 201: 217-231.

Ouyang H, Mou L, Luk C, Liu N, Karaskova J, Squire J, Tsao MS (2000) Immortal human pancreatic duct epithelial cell lines with near normal genotype and phenotype. Am J Pathol 157: 1623-1631.

Poss KD, Wilson LG, Keating MT (2002) Heart regeneration in zebrafish. Science 298: 2188-2190.

Salvatore G, Nappi TC, Salerno P, Jiang Y, Garbi C, Ugolini C, Miccoli P, Basolo F, Castellone MD, Cirafici AM, Melillo RM, Fusco A, Bittner ML, Santoro M (2007) A cell proliferation and chromosomal instability signature in anaplastic thyroid carcinoma. Cancer Res 67: 10148-10158.

Sato N, Fukushima N, Maitra A, Iacobuzio-Donahue CA, van Heek NT, Cameron JL, Yeo CJ, Hruban RH, Goggins M (2004) Gene expression profiling identifies genes associated with invasive intraductal papillary mucinous neoplasms of the pancreas. Am J Pathol 164: 903-914.

Stucke VM, Sillje HH, Arnaud L, Nigg EA (2002) Human Mps1 kinase is required for the spindle assembly checkpoint but not for centrosome duplication. EMBO J 21: 1723-1732.

Taniguchi S, Iwamura T, Katsuki T (1992) Correlation between spontaneous metastatic potential and type I collagenolytic activity in a human pancreatic cancer cell line (SUIT-2) and sublines. Clin Exp Metastasis 10: 259-266. 
Tao Y, Chai D, Ma L, Zhang T, Feng Z, Cheng Z, Wu S, Qin Y, Lai M (2012) Identification of distinct gene expression profiles between esophageal squamous cell carcinoma and adjacent normal epithelial tissues. Tohoku J Exp Med 226: 301-311.

Tardif KD, Rogers A, Cassiano J, Roth BL, Cimbora DM, McKinnon R, Peterson A, Douce TB, Robinson R, Dorweiler I, Davis T, Hess MA, Ostanin K, Papac DI, Baichwal V, McAlexander I, Willardsen JA, Saunders M, Christophe H, Kumar DV, Wettstein DA, Carlson RO, Williams BL (2011) Characterization of the cellular and antitumor effects of MPI-0479605, a small-molecule inhibitor of the mitotic kinase Mps1. Mol Cancer Ther 10: 2267-2275.

Thompson SL, Compton DA (2010) Proliferation of aneuploid human cells is limited by a p53-dependent mechanism. J Cell Biol 188: 369-381.
Toledo LI, Murga M, Gutierrez-Martinez P, Soria R, Fernandez-Capetillo O (2008) ATR signaling can drive cells into senescence in the absence of DNA breaks. Genes Dev 22: 297-302.

Yuan B, Xu Y, Woo JH, Wang Y, Bae YK, Yoon DS, Wersto RP, Tully E, Wilsbach K, Gabrielson E (2006) Increased expression of mitotic checkpoint genes in breast cancer cells with chromosomal instability. Clin Cancer Res 12: 405-410.

This work is published under the standard license to publish agreement. After 12 months the work will become freely available and the license terms will switch to a Creative Commons AttributionNonCommercial-Share Alike 3.0 Unported License.

Supplementary Information accompanies this paper on British Journal of Cancer website (http://www.nature.com/bjc) 